\title{
Evaluation of Xerostomia in Patients with Head and Neck Squamous Cell Carcinoma Treated with Radiotherapy
}

\author{
Aya Hossam Mansy ${ }^{1}$, Alsiagy A. Salama ${ }^{2}$, Fatma Gharib Khair-Allah", \\ Lamiss Mohamed Abd El-Aziz ${ }^{1}$ \\ ${ }^{1}$ Department of Clinical Oncology and Nuclear Medicine, Faculty of Medicine, Tanta University, Tanta, Egypt \\ ${ }^{2}$ Department of Diagnostic Radiology, Faculty of Medicine, Tanta University, Tanta, Egypt \\ Email: ahossam38@gmail.com,siagyali33@yahoo.com,dfatma1980@yahoo.com,lamissmohamed2@yahoo.com, \\ lamismohamed@med.tanta.edu.eg
}

How to cite this paper: Mansy, A.H., Salama, A.A., Khair-Allah, F.G. and El-Aziz, L.M.A. (2020) Evaluation of Xerostomia in Patients with Head and Neck Squamous Cell Carcinoma Treated with Radiotherapy. Journal of Cancer Therapy, 11, 98-114. https://doi.org/10.4236/jct.2020.112009

Received: January 12, 2020

Accepted: February 25, 2020

Published: February 28, 2020

Copyright (c) 2020 by author(s) and Scientific Research Publishing Inc. This work is licensed under the Creative Commons Attribution-NonCommercial International License (CC BY-NC 4.0). http://creativecommons.org/licenses/by-nc/4.0/

\begin{abstract}
Background: The sixth most prevalent cancer in the world is head and neck squamous cell carcinoma (HNSCC). In multiple combinations, surgery, radiation and chemotherapy are used in HNC control. As radiation-induced salivary gland damage and xerostomia is one of the most usual and distressing impacts, diffusion-weighted magnetic resonance imaging (DW-MRI) is a promising technique for the assessment of alterations due to radiation therapy. The aim of the study is to evaluate the effect of Chemoradiotherapy either concurrent or sequential/RTH on salivary glands using DW-MRI performed before and after chemoradiotherapy/RTH, correlation between DW-MRI changes and delivered radiation dose to salivary glands, study the acute toxicity of chemoradiotherapy/RTH on salivary glands and assess quality of life for patients with radiation-induced xerostomia. Patients and Methods: This prospective study included 43 patients with Head and Neck squamous cell carcinoma treated with definitive radiotherapy, sequential therapy or concurrent chemoradiotherapy at Clinical Oncology and Nuclear Medicine Department, MRI diffusion scans were done at Diagnostic Radiology Department Tanta University Hospitals throughout the period from May 2016 to May 2019. DW-MRI performed before and after RTH. For patients receiving CCRTH, DW sequence will be performed before starting RTH and 2 - 3 months post-RTH once at rest and then repeated continuously during salivary stimulation by ascorbic acid. In case of induction chemotherapy, DW-MRI was done before and after induction and 2 - 3 months post-RTH. Results: In all time periods, ADC values were lower for the PG than the SMG. ADC change of PGs was higher in hypopharyngeal carcinoma, while ADC change of SMGs
\end{abstract}


were higher in oropharyngeal carcinoma. ADC change in PGs \& SMGs was higher in advanced stage. Conclusion: To assess the radiation-induced xerostomia, DW-MRI can be used as non-invasive tool. Xerostomia questionnaire is helpful instrument for evaluating of quality of life for patients with radiation-induced xerostomia. There was a significant correlation between xerostomia and quality of life.

\section{Keywords}

Xerostomia, Diffusion-Weighted MRI, Radiotherapy, Toxicity

\section{Background}

Squamous cell carcinoma of the head and neck (HNSCC) is the world's sixth most common cancer [1].

For early stage HNSCC, patient achieved complete response when either treated with either surgery or radiotherapy. While, locally advanced HNSCC requires aggressive multimodality therapy incorporating local and systemic therapy with either chemotherapy and/or targeted therapy [2].

Although many patients are being cured of their disease, a significant percentage of survivors are suffering from the adverse effects associated with treatment. Head and neck cancer and its treatment may affect the quality of life related to disease specific health (e.g., salivary and swallowing functions) [3].

Damage to the salivary gland and xerostomia caused by radiation is one of the most prevalent and distressing adverse effects. xerostomia is probably caused by several pronounced variables, such as radiation dose distribution, demographics, tumors and variables linked to treatment [4].

The extent of the dysfunction is associated with the dose and the volume of salivary tissue irradiated [5].

Minimal dysfunction of the gland can be noted at mean doses of 10 to $15 \mathrm{~Gy}$ and mean doses of $>40 \mathrm{~Gy}$ to the parotid can lead to a decrease in function of 75 percent, the dysfunction at doses $>54 \mathrm{~Gy}$ is regarded irreversible [6].

It is of great importance to early detect and prevent xerostomia. Thus far, salivary gland scintigraphy (SGS) and quantitative salivary flow rate measurements have been the cornerstone of evaluating salivary gland function in HNC patients SGS has been shown to be viable for anticipating salivary gland function after radiation therapy (RTH), but the usefulness of this method of measurement is restricted by its invasiveness and the radiation exposure associated with it [7].

In patients with HNSCC, a diffusion-weighted MRI (DWI) is frequently done. Even during the early phases of the disease, the parameters of the apparent diffusion coefficient (ADC) may represent tissue microstructures or path physiological circumstances. Certainly, the ADC values could represent salivary gland function and xerostomia following RTH [8]. 


\section{Aim}

Study the effect of chemoradiotherapy either concurrent or sequential/RTH on salivary glands using DW-MRI performed before and after chemoradiotherapy/RTH, correlation between DW-MRI changes and delivered radiation dose to salivary glands, study the acute toxicity of chemoradiotherapy/RTH on salivary glands and assess quality of life for patients with radiation induced xerostomia.

\section{Patients and Methods}

This prospective study included 43 patients with Head and Neck squamous cell carcinoma treated with definitive radiotherapy, sequential therapy or concurrent chemoradiotherapy at Clinical Oncology and Nuclear Medicine Department, MRI diffusion scans were done at Diagnostic Radiology Department, throughout the period from May 2016 to May 2019.

Inclusion criteria: Patients with early or locally advanced HNSCC staged according TNM $8^{\text {th }}$ edition [9] aged more than 18 years old of ECOG performance status 0 - 2 with adequate hematological tests, renal and hepatic functions.

Exclusion criteria: Patient with clinical hearing loss, cardiac dysfunction, systemic disease of salivary glands, other comorbidities \& uncooperative patients.

Pre-treatment evaluation for all patients was done including: Complete history, clinical examination, routine laboratory investigations, radiological imaging tumor pathology and staging and Pre-treatment dental care.

Forty three patients were evaluated for xerostomia using DW-MRI, fifteen patients with early glottic scc received definitive RTH (60 - 66 Gy), while patients with locally advanced scc divided into 9 patients received sequential therapy and 19 patients received CCRTH.

Patients with glottic laryngeal SCC received 60 - 66 Gy, 2 Gy/fraction while patients with locally advanced laryngeal, hypopharyngeal and oropharyngeal SCC received radiotherapy in dose of $70 \mathrm{~Gy}$ in 35 fractions over 7 weeks with sequential therapy or CCRTH.

All patients had a contrast enhanced planning computed tomography (CT) scan (2.5 mm slice thickness) in an immobilization thermoplastic mask. Patient set-up supine and head position was hyperextended, clinically palpable LNs were outlined with metallic wire and in cases of oral cavity a tongue blade used to depress the tongue.

Treatment was performed using the Eclipse treatment planning system (Varian Medical Systems). Closed high speed MRI machine (General electric SIGNA) 1.5 TESLA. Dynamic contrast MRI was done by two dimensional fast spoiled gradient recalled echo with fat suppression in T1WI (TR $4.3 \mathrm{~ms}$, TE $1.3 \mathrm{~ms}$ ). MRI DW-MRI performed in 20 patients with HNC before and after RTH. For patients receiving CCRTH, DW sequence will be performed before starting RTH and 2 - 3 months post-RTH once at rest and then repeated continuously during salivary stimulation by ascorbic acid. In case of induction chemotherapy, DW-MRI was done before and after induction and 2 - 3 months post-RTH. 
Ten spherical regions of interest $7 \mathrm{~mm}$ in diameter (ROIs) defined for each patient on the planning CT scan: three in each PG (upper part, lower part and deep part) and two in each SMG (anterior and posterior). For each patient ADC values for both PG and SMG calculated.

For all examinations, a spine and neck coil head coil combined with a phased array was used. TR/TE $=5000 / 105 \mathrm{~ms}$, in-plane pixel size $=2 \times 2 \mathrm{~mm}$, and $\mathrm{b}$ values $=0,500$ and $1000 \mathrm{~s} / \mathrm{mm}^{2}$ (three averages) were echo planar images parameters.

\section{Patient Assessment}

\subsection{Assessment of Common Toxicity}

Any adverse events were recorded. Chemotherapy toxicity grading was based on the common terminology criteria for adverse event ((NCI-CTC 5.0) [10]. acute and late toxicity criteria of the Radiation Therapy Oncology Group (RTOG) were used for grading radiotherapy toxicity [11].

\subsection{Assessment of Quality of Life Related Xerostomia}

Quality of life related xerostomia was based on (XQ of University of Michigan \& QoL questionnaire by Dirix et al. [12].

\subsection{Statistical Analysis}

IBM SPSS Statistics software package version 21 was used for data analysis. Quantitative data was expressed using range, mean and standard deviation while Qualitative data was expressed in frequency and percent. Qualitative data was analyzed using Monte Carlo test. p-value was assumed to be significant at 0.05 [13].

\section{Results}

Age ranged from 40 to 70 years with mean age was 56 years with SD $+9.2,50 \%$ were males and $48.8 \%$ were smokers.

Most patients were locally advanced SCC (65\%) while (35\%) were early stage. Twenty six patients presented with laryngeal carcinoma (60.5\%) from whom fifteen patients were glottic \& eleven patients were supraglottic, while $23.3 \%$ presented with oropharyngeal carcinoma and $13.2 \%$ presented with hypopharyngeal carcinoma. Most patients were of performance 0/1. Patients' characteristics were shown in Table 1.

There was significant increase in ADC values for parotid glands post RTH post stimulation and this was also significant in pre-stimulation as shown in $\mathrm{Ta}$ ble $2 \&$ as regard submandibular glands, it was also the same as shown in Table 3 and Figure $1 \&$ Figure 2.

As regard sequential group, there was an increase of ADC value post stimulation post-CTH for all ROI of parotid gland, but this increase was not significant 
Table 1. Socio-demographic and clinical characteristics of 43 patients with HNSCC.

\begin{tabular}{|c|c|}
\hline Item & Number \% \\
\hline \multicolumn{2}{|l|}{ Gender } \\
\hline Male & $21(48.8 \%)$ \\
\hline Female & $22(51.2 \%)$ \\
\hline \multicolumn{2}{|l|}{ Age } \\
\hline Range (years) & $40-70$ \\
\hline Mean \pm SD & $55.9 \pm 9.2$ \\
\hline \multicolumn{2}{|l|}{ Smoking } \\
\hline Yes & $21(48.8 \%)$ \\
\hline No & $22(51.2 \%)$ \\
\hline \multicolumn{2}{|l|}{ Performance status } \\
\hline $0 / 1$ & $28(65.1 \%)$ \\
\hline 2 & $15(34.9 \%)$ \\
\hline \multicolumn{2}{|l|}{ Sites } \\
\hline Larynx & $26(60.5 \%)$ \\
\hline Oropharynx & $10(23.3 \%)$ \\
\hline Hypopharynx & $7(16.2 \%)$ \\
\hline \multicolumn{2}{|l|}{ Subsites } \\
\hline Glottic & $15(34.9 \%)$ \\
\hline Supraglottic & $11(25.6 \%)$ \\
\hline Tongue & $11(25.6 \%)$ \\
\hline Post cricoid & $6(13.9 \%)$ \\
\hline \multicolumn{2}{|l|}{ Tumor size } \\
\hline $\mathrm{T} 1$ & $0(0 \%)$ \\
\hline $\mathrm{T} 2$ & $21(48.8 \%)$ \\
\hline T3 & $17(39.6 \%)$ \\
\hline $\mathrm{T} 4$ & $5(11.6 \%)$ \\
\hline \multicolumn{2}{|l|}{ Lymph nodes } \\
\hline No & $15(34.9 \%)$ \\
\hline N1 & $15(34.9 \%)$ \\
\hline $\mathrm{N} 2$ & $9(20.9 \%)$ \\
\hline N3 & $4(9 \% .3)$ \\
\hline \multicolumn{2}{|l|}{ Stage } \\
\hline II & $15(34.9 \%)$ \\
\hline III & $9(20.9 \%)$ \\
\hline Iva\&b & $19(44.1 \%)$ \\
\hline \multicolumn{2}{|l|}{ Treatment modalities } \\
\hline Definitive radiotherapy & $15(34.9 \%)$ \\
\hline Induction chemotherapy followed by CCRTH & $9(20.9 \%)$ \\
\hline CCRTH & $19(44.1 \%)$ \\
\hline
\end{tabular}

except for LT lower lobe as shown in Table 4 \& as regard SMG, it was the same but the increase was not significant as shown in Table 5.

Mean RTH doses delivered to RT \& LT parotid were 34.67 Gy \& 34 Gy rspectively while mean doses delivered to RT \& LT SMG were 41 Gy \& 40 Gy respectively as shown in Table 6. 
Table 2. Post-stimulation ADC values for PGs in pre \& post radiotherapy.

\begin{tabular}{|c|c|c|c|c|c|c|c|c|c|c|}
\hline \multirow{2}{*}{ Parotid gland } & \multicolumn{4}{|c|}{$\begin{array}{c}\text { ADC pre RTH } \\
\text { post Stimulation }\end{array}$} & \multicolumn{4}{|c|}{$\begin{array}{l}\text { ADC post RTH } \\
\text { post Stimulation }\end{array}$} & \multicolumn{2}{|c|}{ T-test } \\
\hline & $\mathrm{N}$ & Mean & \pm & $\mathrm{SD}$ & $\mathrm{N}$ & Mean & \pm & SD & $\mathrm{t}$ & $\mathrm{p}$-value \\
\hline RT Upper lobe & 43 & 75.55 & \pm & 6.92 & 43 & 88.15 & \pm & 10.41 & 4.506 & $<0.001^{\star}$ \\
\hline RT Lower lobe & 43 & 85.40 & \pm & 7.73 & 43 & 101.65 & \pm & 12.36 & 4.987 & $<0.001^{\star}$ \\
\hline RT Deep lobe & 43 & 84.35 & \pm & 8.39 & 43 & 99.40 & \pm & 10.74 & 4.938 & $<0.001^{\star}$ \\
\hline LT Upper lobe & 43 & 74.65 & \pm & 7.65 & 43 & 91.50 & \pm & 8.30 & 6.675 & $<0.001^{\star *}$ \\
\hline LT Lower lobe & 43 & 86.15 & \pm & 6.43 & 43 & 104.30 & \pm & 13.24 & 5.514 & $<0.001^{\star}$ \\
\hline LT Deep lobe & 43 & 85.15 & \pm & 8.42 & 43 & 102.05 & \pm & 11.18 & 5.399 & $<0.001^{*}$ \\
\hline
\end{tabular}

*Statistical significant.

Table 3. Post-stimulation ADC values for SMGs in pre \& post radiotherapy.

\begin{tabular}{ccccccccccccc}
\hline \multirow{2}{*}{$\begin{array}{c}\text { Sub mandibular } \\
\text { gland }\end{array}$} & \multicolumn{3}{c}{$\begin{array}{c}\text { ADC pre RTH } \\
\text { post Stimulation }\end{array}$} & \multicolumn{3}{c}{$\begin{array}{c}\text { ADC Post RTH } \\
\text { Post Stimulation }\end{array}$} & & T-test \\
\cline { 2 - 12 } & $\mathrm{N}$ & Mean & \pm & SD & N & Mean & \pm & SD & t & p-value \\
\hline RT Anterior lobe & 43 & 95.35 & \pm & 10.22 & 43 & 114.95 & \pm & 11.66 & 5.652 & $<0.001^{*}$ \\
RT Posterior lobe & 43 & 96.10 & \pm & 12.05 & 43 & 115.75 & \pm & 8.99 & 5.846 & $<0.001^{*}$ \\
LT Anterior lobe & 43 & 96.60 & \pm & 10.73 & 43 & 119.05 & \pm & 9.46 & 7.021 & $<0.001^{*}$ \\
LT Posterior lobe & 43 & 97.30 & \pm & 11.33 & 43 & 118.40 & \pm & 9.45 & 6.395 & $<0.001^{*}$ \\
\hline * Statistical significant. & & & & & & & & & &
\end{tabular}

Table 4. Post-stimulation ADC values for PGs in pre-RTH \& pre-chemotherapy.

\begin{tabular}{|c|c|c|c|c|c|c|c|c|c|c|}
\hline \multirow[t]{2}{*}{ Parotid gland } & \multicolumn{4}{|c|}{$\begin{array}{c}\text { ADC } \\
\text { Pre chemotherapy } \\
\text { Post stimulation }\end{array}$} & \multicolumn{4}{|c|}{$\begin{array}{c}\text { ADC } \\
\text { Pre RTH } \\
\text { Post stimulation }\end{array}$} & \multicolumn{2}{|c|}{ T-test } \\
\hline & $\mathrm{N}$ & Mean & \pm & $\mathrm{SD}$ & $\mathrm{N}$ & Mean & \pm & SD & $\mathrm{t}$ & p-value \\
\hline RT Upper lobe & 9 & 70.38 & \pm & 9.38 & 9 & 76.50 & \pm & 9.06 & 1.329 & 0.205 \\
\hline RT Lower lobe & 9 & 79.88 & \pm & 8.81 & 9 & 86.38 & \pm & 9.33 & 1.433 & 0.174 \\
\hline RT Deep lobe & 9 & 77.75 & \pm & 9.16 & 9 & 83.50 & \pm & 9.27 & 1.248 & 0.233 \\
\hline LT Upper lobe & 9 & 68.50 & \pm & 7.35 & 9 & 75.63 & \pm & 11.06 & 1.518 & 0.151 \\
\hline LT Lower lobe & 9 & 75.88 & \pm & 4.36 & 9 & 86.00 & \pm & 8.55 & 2.984 & $0.010^{*}$ \\
\hline LT Deep lobe & 9 & 77.00 & \pm & 8.11 & 9 & 85.88 & \pm & 10.91 & 1.847 & 0.086 \\
\hline
\end{tabular}

*Statistical significant.

Table 5. Post-stimulation ADC values for SMGs for pre-RTH \& pre-chemotherapy in sequential group (9 patients who received sequential treatment).

\begin{tabular}{|c|c|c|c|c|c|c|c|c|c|c|}
\hline \multirow[t]{2}{*}{$\begin{array}{l}\text { Sub mandibular } \\
\text { gland }\end{array}$} & \multicolumn{4}{|c|}{$\begin{array}{c}\text { ADC } \\
\text { Pre chemotherapy } \\
\text { Post stimulation }\end{array}$} & \multicolumn{4}{|c|}{$\begin{array}{c}\text { ADC } \\
\text { Pre RTH } \\
\text { Post stimulation }\end{array}$} & \multicolumn{2}{|c|}{ T-test } \\
\hline & $\mathrm{N}$ & Mean & \pm & $\mathrm{SD}$ & $\mathrm{N}$ & Mean & \pm & SD & $\mathrm{t}$ & $\mathrm{p}$-value \\
\hline RT Anterior lobe & 9 & 87.38 & \pm & 10.85 & 9 & 93.88 & \pm & 12.57 & 1.107 & 0.287 \\
\hline RT Posterior lobe & 9 & 87.00 & \pm & 9.50 & 9 & 97.13 & \pm & 13.35 & 1.748 & 0.102 \\
\hline LT Anterior lobe & 9 & 86.63 & \pm & 11.61 & 9 & 96.63 & \pm & 14.03 & 1.553 & 0.143 \\
\hline LT Posterior lobe & 9 & 89.25 & \pm & 11.32 & 9 & 98.88 & \pm & 14.47 & 1.482 & 0.161 \\
\hline
\end{tabular}




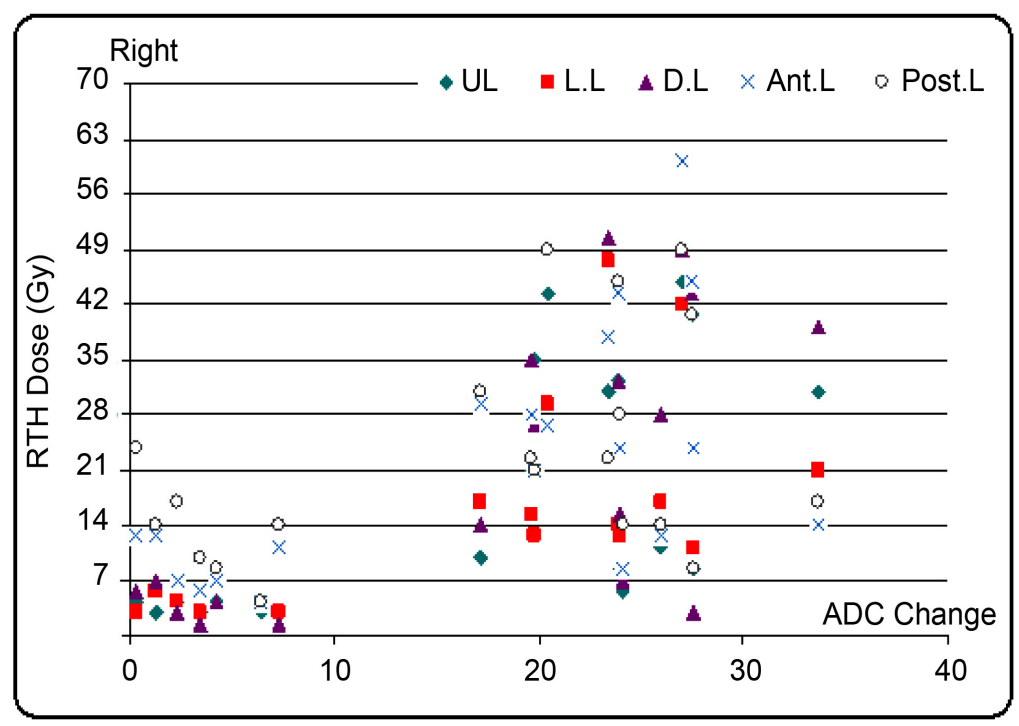

Figure 1. Change in DW-MRI ADC values of RT PGs \& SMGs 3 months after therapy versus radiotherapy dose.

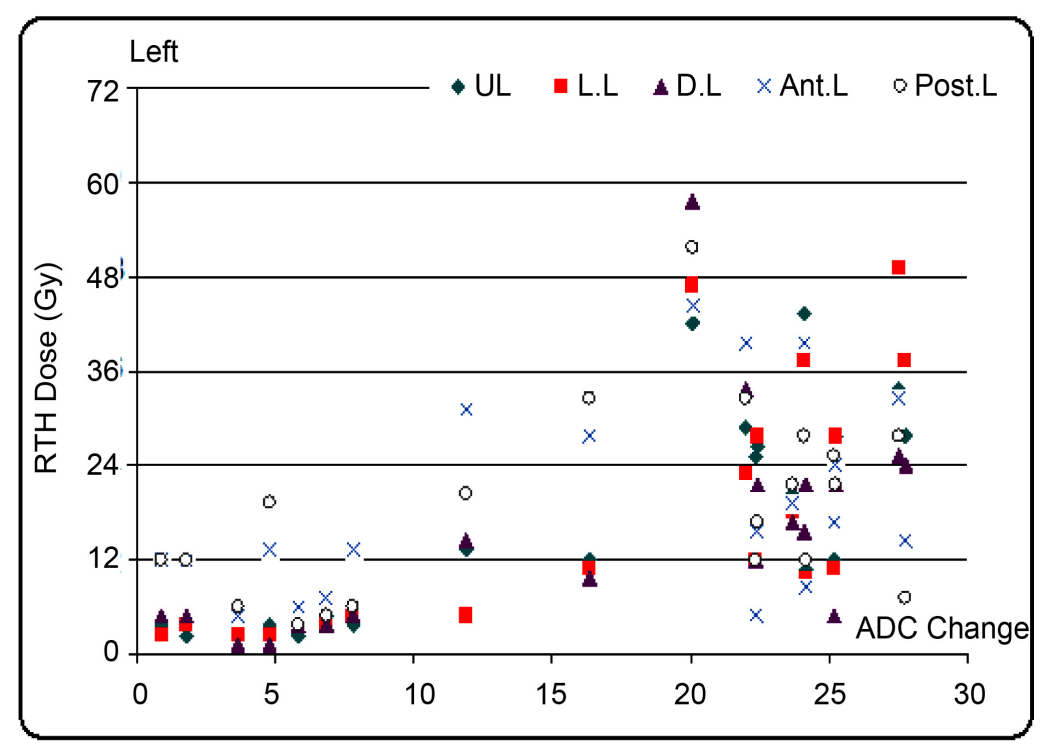

Figure 2. Change in DW-MRI ADC values of LT PGs \& SMGs 3 months after therapy versus radiotherapy dose.

Table 6. Radiotherapy doses delivered to salivary glands.

\begin{tabular}{|c|c|}
\hline \multicolumn{2}{|c|}{ Radiotherapy dose } \\
\hline$R T$ Parotid & \\
\hline Mean \pm SD & $34.67 \pm 25.82$ \\
\hline \multicolumn{2}{|l|}{ LT Parotid } \\
\hline Mean \pm SD & $33.90 \pm 27.97$ \\
\hline \multicolumn{2}{|c|}{$R T$ Submandibular } \\
\hline Mean \pm SD & $40.92 \pm 27.92$ \\
\hline \multicolumn{2}{|c|}{ LT Submandibular } \\
\hline Mean \pm SD & $39.86 \pm 28.22$ \\
\hline
\end{tabular}


In case of early glottic SCC, RTH dose delivered to RT Parotid \& SMG was low and this correlated with low ADC changes while in cases of locally advanced stage, radiotherapy dose delivered to RT parotid \& SMG was high and this correlated with marked changes in ADC values as shown in Table 2, and it was the same as regard LT parotid and LT SMG as shown in Table 3.

ADC change was statistically significant with site, stage and treatment modality, so high grade xerostomia was noticed in patients with locally advanced HNSCC treated with sequential therapy as shown in Table 7, Table 8, Case 1 \& Case 2.

Table 7. Correlation between ADC changes and clinical characteristics.

\begin{tabular}{|c|c|c|c|c|}
\hline & $\begin{array}{c}\text { Parotid } \\
\text { ADC change } \\
(\text { Mean } \pm \mathrm{SD})\end{array}$ & $\begin{array}{l}\text { Sub mandibular } \\
\text { ADC change } \\
\text { (Mean } \pm \mathrm{SD})\end{array}$ & $x^{2}$ & $\mathrm{p}$-value \\
\hline \multicolumn{5}{|l|}{ Site } \\
\hline Larynx & $14.64 \pm 12.73$ & $15.55 \pm 12.34$ & \multirow{3}{*}{15.62} & \multirow{3}{*}{$0.018^{*}$} \\
\hline Oropharynx & $14.53 \pm 10.21$ & $18.06 \pm 11.45$ & & \\
\hline Hypopharynx & $15.77 \pm 4.63$ & $17.25 \pm 5.69$ & & \\
\hline \multicolumn{5}{|l|}{ Sub site } \\
\hline Glottic & $14.32 \pm 11.98$ & $15.7 \pm 13.24$ & \multirow{4}{*}{12.86} & \multirow{4}{*}{$0.021^{\star}$} \\
\hline Supraglottic & $15.11 \pm 12.36$ & $14.44 \pm 12.04$ & & \\
\hline Tongue & $14.56 \pm 10.10$ & $18.06 \pm 14.12$ & & \\
\hline Post cricoids & $15.77 \pm 2.64$ & $17.25 \pm 5.66$ & & \\
\hline \multicolumn{5}{|l|}{ Performance } \\
\hline $0 / 1$ & $12.23 \pm 10.62$ & $13.02 \pm 11.42$ & \multirow[t]{2}{*}{6.88} & \multirow[t]{2}{*}{0.441} \\
\hline 2 & $14.87 \pm 11.58$ & $15.62 \pm 12.57$ & & \\
\hline \multicolumn{5}{|l|}{ Stage } \\
\hline II & $11.58 \pm 9.33$ & $13.25 \pm 10.96$ & \multirow{3}{*}{11.21} & \multirow{3}{*}{0.054} \\
\hline III & $14.04 \pm 10.29$ & $15.02 \pm 13.32$ & & \\
\hline IV & $9.18 \pm 7.86$ & $10.35 \pm 8.76$ & & \\
\hline \multicolumn{5}{|l|}{ T stage } \\
\hline T2 & $10.23 \pm 8.69$ & $12.85 \pm 9.89$ & \multirow{3}{*}{10.00} & \multirow{3}{*}{$0.042^{\star}$} \\
\hline $\mathrm{T} 3$ & $13.52 \pm 11.46$ & $15.21 \pm 12.85$ & & \\
\hline $\mathrm{T} 4$ & $12.36 \pm 10.68$ & $12.79 \pm 10.56$ & & \\
\hline \multicolumn{5}{|l|}{$\mathrm{N}$ stage } \\
\hline No & $12.48 \pm 10.36$ & $14.36 \pm 11.55$ & \multirow{4}{*}{6.88} & \multirow{4}{*}{$0.031^{\star}$} \\
\hline N1 & $14.75 \pm 12.31$ & $15.91 \pm 12.89$ & & \\
\hline $\mathrm{N} 2$ & $15.29 \pm 12.64$ & $15.88 \pm 9.88$ & & \\
\hline N3 & $11.57 \pm 9.82$ & $12.24 \pm 10.23$ & & \\
\hline
\end{tabular}

*Statistical significant.

Table 8. Correlation between ADC changes and treatment modality.

\begin{tabular}{cccccc}
\hline & $\begin{array}{c}\text { Sequential } \\
(\mathrm{n}=8)\end{array}$ & $\begin{array}{c}\text { CCRTH } \\
(\mathrm{n}=17)\end{array}$ & $\begin{array}{c}\text { Definitive } \\
(\mathrm{n}=18)\end{array}$ & $\chi^{2}$ & p-value \\
\hline RT Parotid & $17.2 \pm 15.6$ & $15.25 \pm 14.31$ & $14.6 \pm 10.97$ & & \\
LT Parotid & $16.6 \pm 12.4$ & $15.23 \pm 12.52$ & $13.26 \pm 11.61$ & 19.22 & $0.006^{*}$ \\
RT Submandibular & $17.23 \pm 14.52$ & $16.38 \pm 13.67$ & $15.18 \pm 12.23$ & & \\
LT Submandibular & $17.19 \pm 13.82$ & $16.24 \pm 13.14$ & $14.14 \pm 11.12$ & & \\
\hline
\end{tabular}

*Statistical significant. 
After completion of radiotherapy, patients were assessed for xerostomia using Michigan University xerostomia questionnaire. Mean XQ values were 53.7 with SD +19.59 (65\% of patients exceed severity criterion XQ > 30). Patients experienced xerostomia were 13 patients $(65 \%)$ while 7 patients $(35 \%)$ were not experiencing xerostomia.

Xerostomia related quality of life scale questionnaire (15 questions), the answer of each question were as follows: $1,2,3,4,5$ : defined as not at all, a little, moderately, quite a lot; and very much respectively. It was ranged between 15 75. The higher the score was, the worse the quality of life.

Patients who had xerostomia with low quality of life, and high quality were reported in 10 patients $(76.9 \%), 3$ patients (23\%). respectively however, low quality of life was reported in one patient (14.3\%) and high quality in 6 patients $(85.7 \%)$ in patients with no xerostomia. among HNSCC receiving RTH statistical significant association between quality of life and xerostomia $\left(\mathrm{p}=0.028^{\star}\right)$ as shown in Table 9.

Case 1 (Figure 3): Male patient aged 65 years old presented with supraglottic with pyriform sinus extension SCC T2N2 M0 was treated with sequential therapy.

Case 2 (Figure 4): Male patient aged 53 years old presented with glottic SCC T2N0 M0 was treated with definitive radiotherapy.

Table 9. Xerostomia and quality of life.

\begin{tabular}{ccccc}
\hline \multirow{2}{*}{ Xerostomia } & \multicolumn{2}{c}{ Quality of life } & & $\mathrm{n}$ \\
\cline { 2 - 4 } & High & Low & & $\mathrm{p}$ \\
\hline Xerostomia $(+)$ & $3(25 \%)$ & $21(75 \%)$ & & \\
Xerostomia $(-)$ & $12(80 \%)$ & $3(20 \%)$ & 15 & $0.028^{*}$ \\
Total & 15 & 24 & 43 & \\
\hline
\end{tabular}

*Statistical significant.

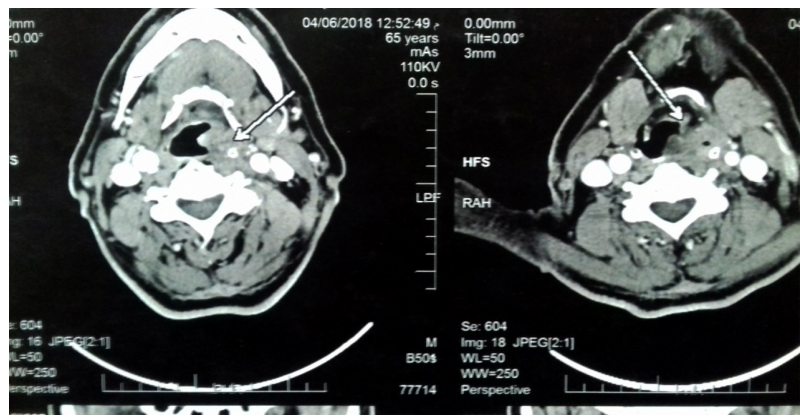

(a)

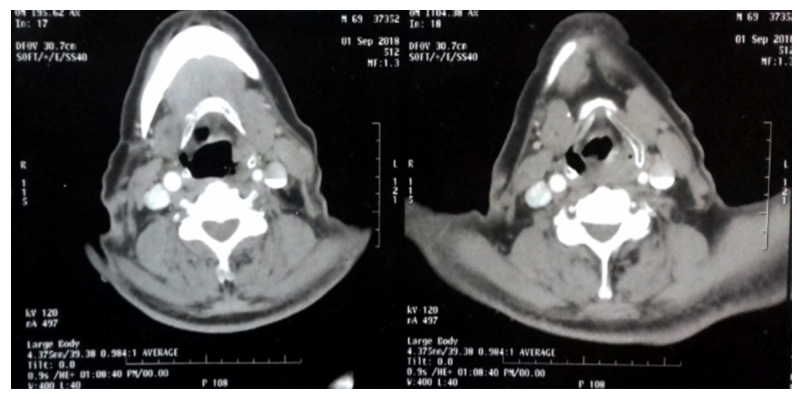

(b) 


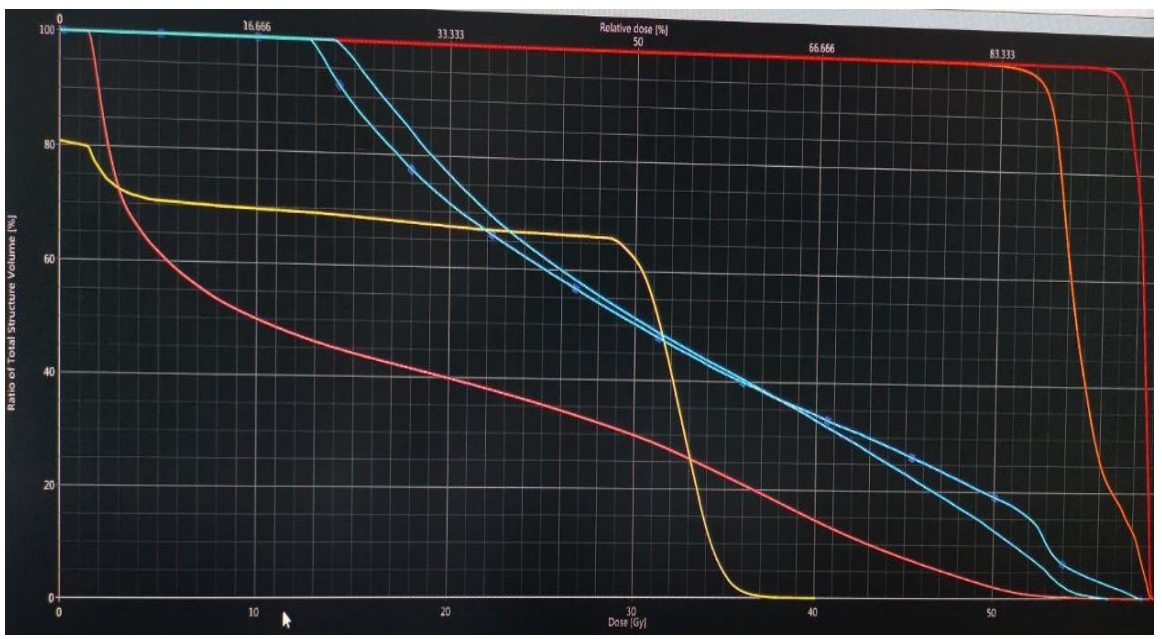

(c)

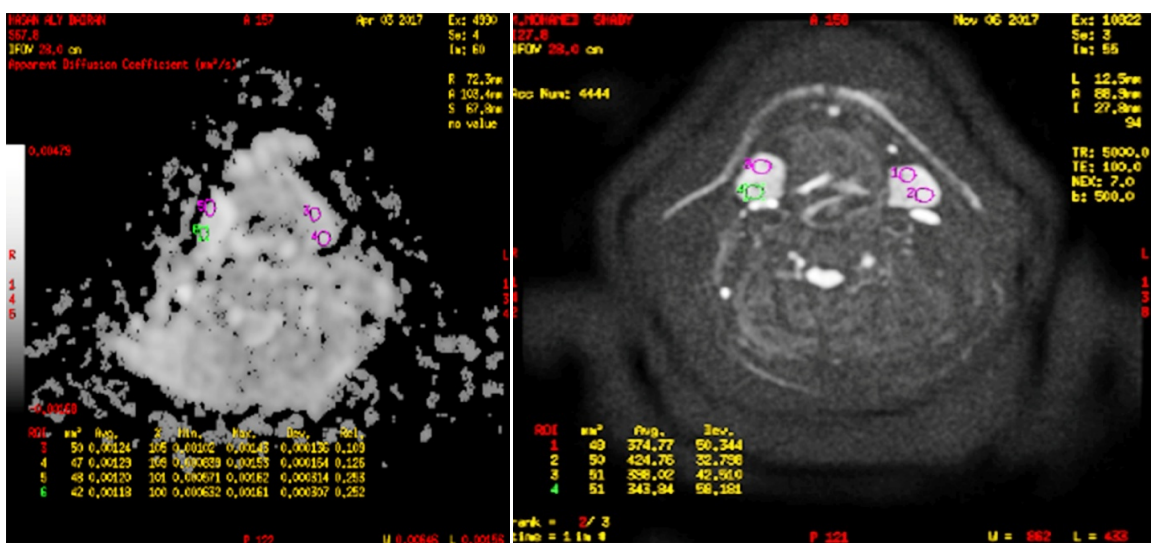

(d)
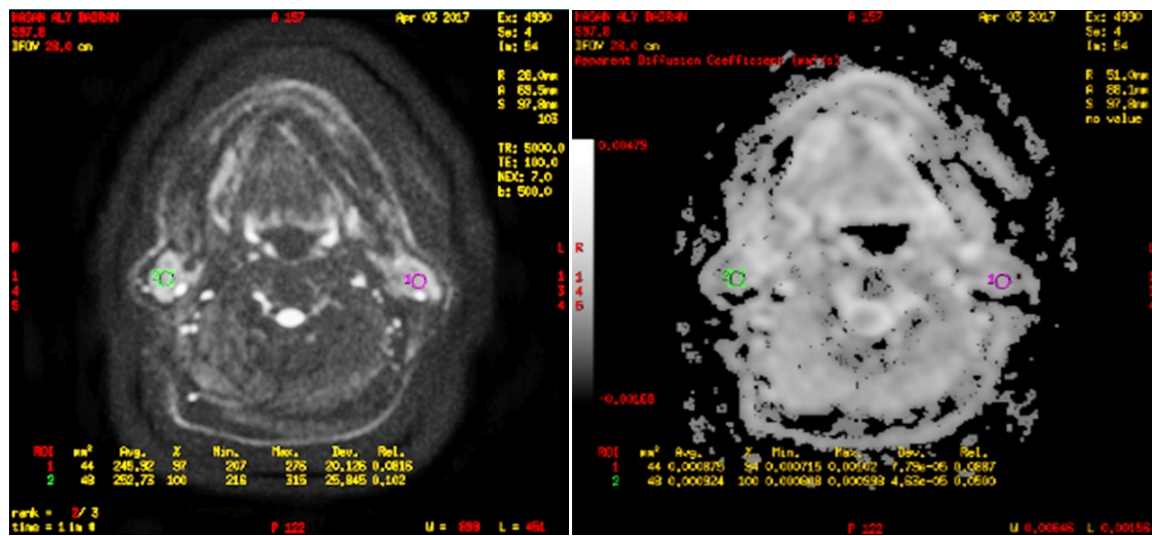

(e)

Figure 3. (a) Initial presentation of CT neck, shows supraglottic and glottic mass with bilateral cervical lymph node; (b) CT neck, one month after chemoradiotherapy end, shows complete response; (c) DVH of salivary glands shows: mean doses of RT \& LT PGs (17.12 Gy \& 17.59 Gy respectively) while mean dose of RT \& LT SMGs are (62 Gy \& 62.7 Gy respectively; (d) MRI diffusion of both SMGs post RTH, showing increased ADC value (post ROI of RT SMG, ADC value: $180 \times 10^{-5} \mathrm{~mm}^{2} / \mathrm{s}$ ) compared with pre-RT ADC value $\left(116 \times 10^{-5} \mathrm{~mm}^{2} / \mathrm{s}\right)$; (e) MRI diffusion of upper lobes of both PGs post RTH, showing increased ADC value (post ROI of RT PG, ADC value: $166 \times 10^{-5} \mathrm{~mm}^{2} / \mathrm{s}$ ) compared with pre-RT ADC value $\left(80 \times 10^{-5} \mathrm{~mm}^{2} / \mathrm{s}\right)$. 

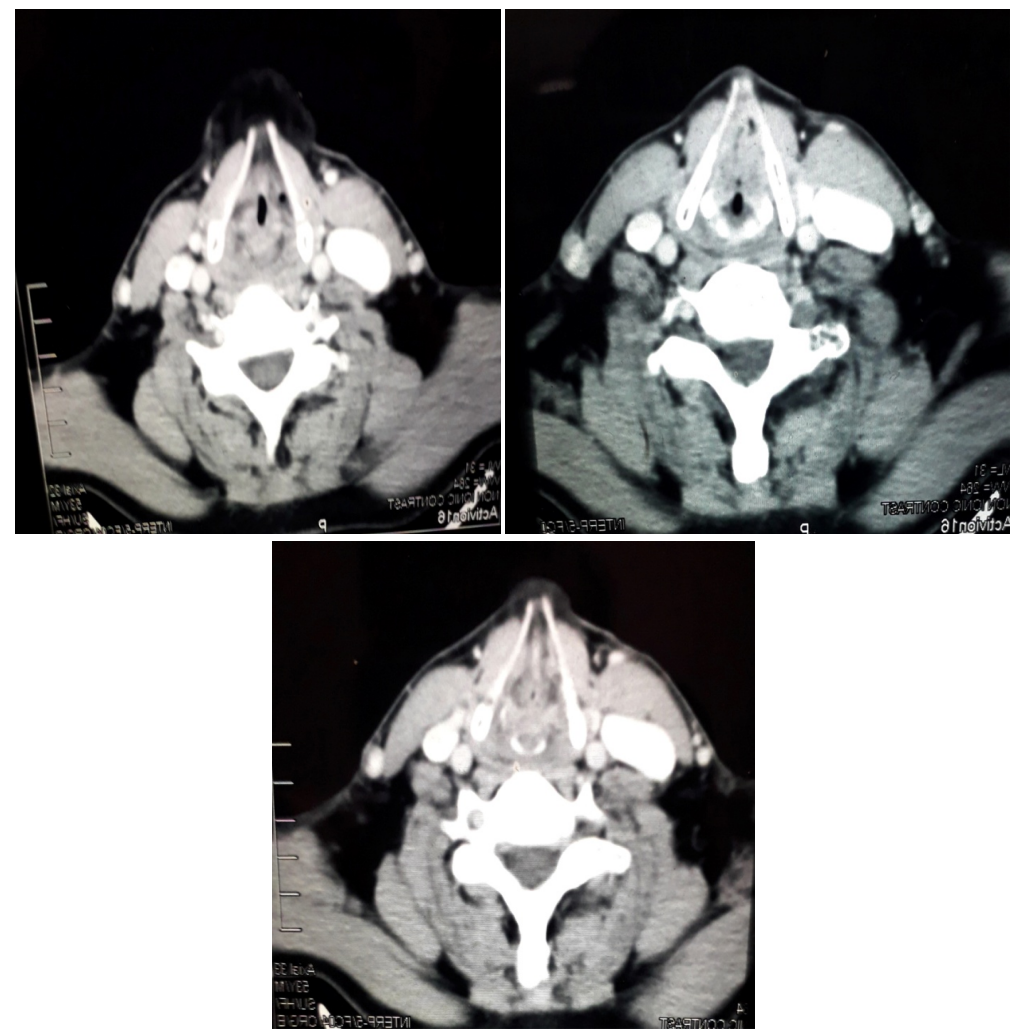

(a)

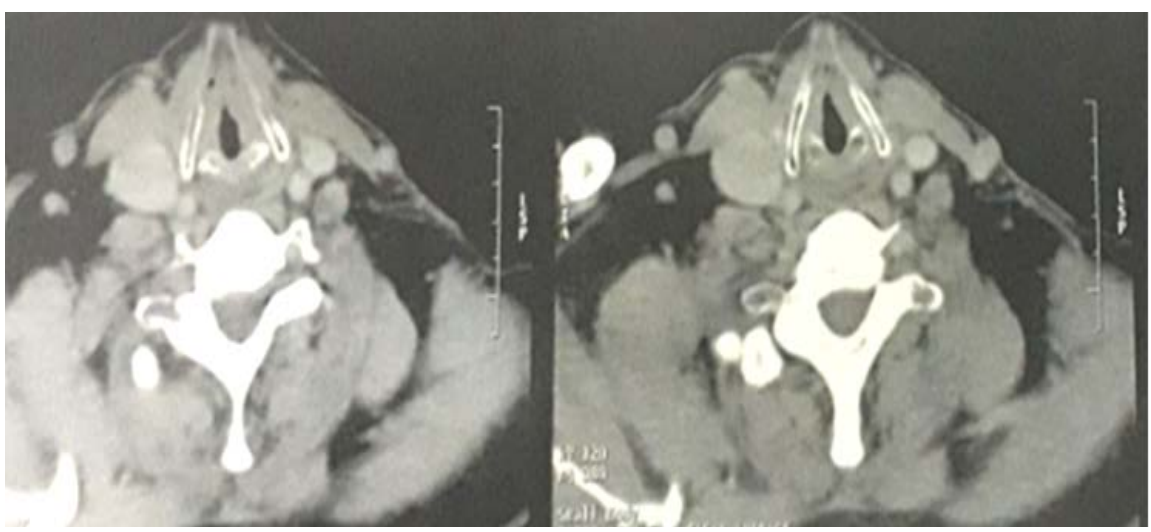

(b)

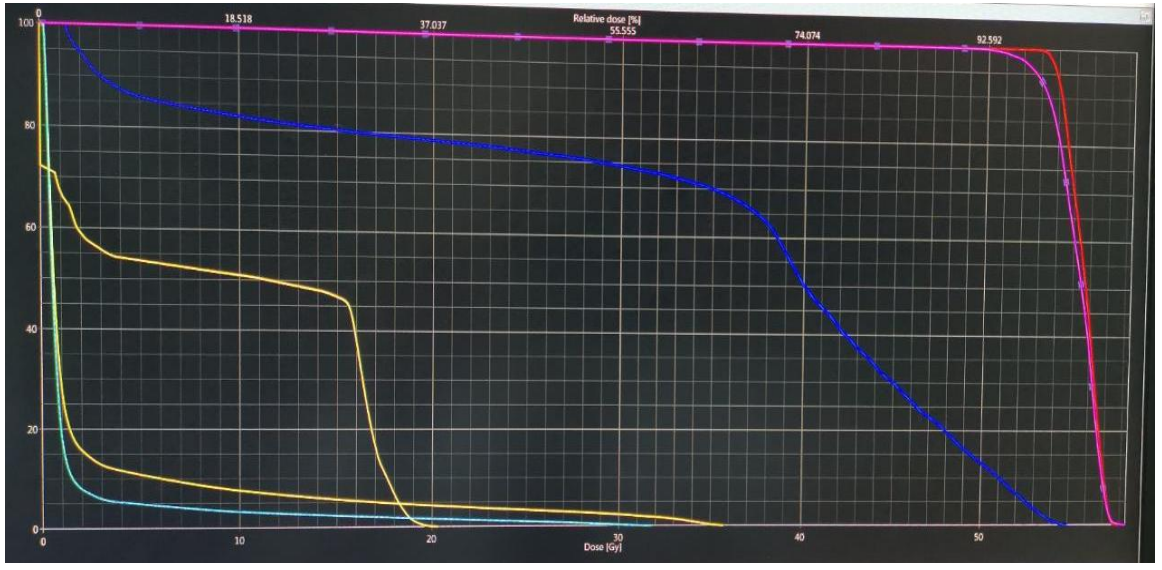

(c) 


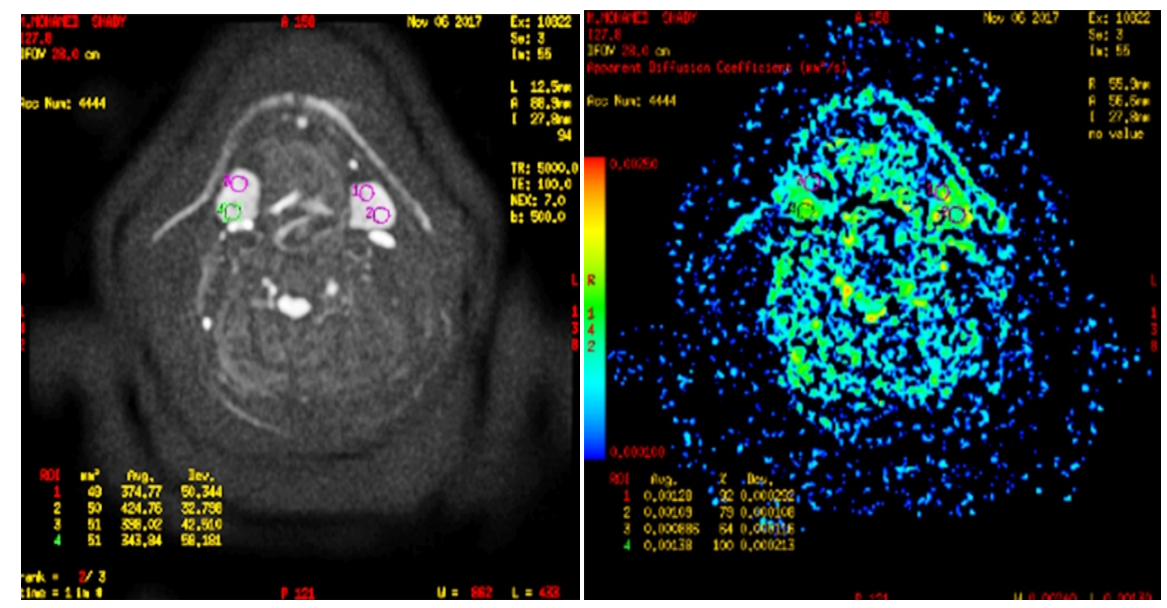

(d)
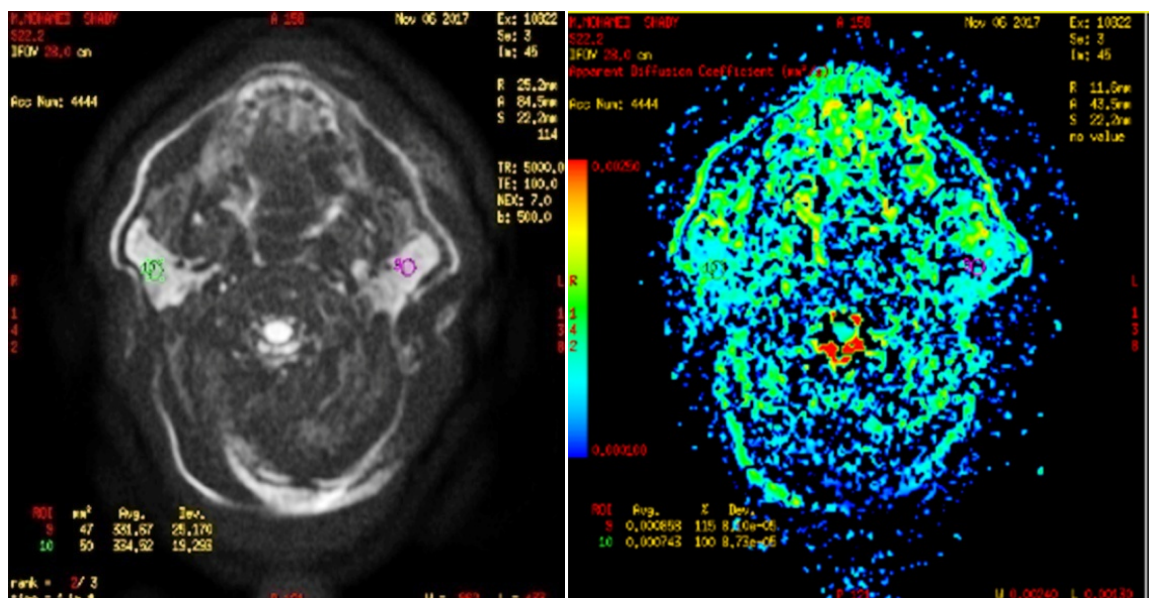

(e)

Figure 4. (a) Initial presentation CT neck, shows localized LT vocal cord mass without supraglottic extension; (b) CT neck, one month after definitive radiotherapy end, shows complete response; (c) DVH of salivary glands mean doses of RT \& Lt PGs $(0.28$ Gy \& 0.29 Gy respectively) while mean doses RT \& LT SMG EARE (1.4 Gy \& 1.5 Gy respectively); (d) MRI diffusion of both SMGs post RTH, showing increased ADC value (post ROI of RT SMG, ADC value: $115 \times 10^{-5} \mathrm{~mm}^{2} / \mathrm{s}$ ) compared with pre-RT ADC value (102 $\times 10^{-5} \mathrm{~mm}^{2} / \mathrm{s}$ ); (e) MRI diffusion of upper lobes of both PGs post RTH, showing increased ADC value (post ROI of RT PG, ADC value: $67 \times 10^{-5} \mathrm{~mm}^{2} / \mathrm{s}$ ) compared with pre-RT ADC value $\left(62 \times 10^{-5} \mathrm{~mm}^{2} / \mathrm{s}\right)$.

\section{Discussion}

In all head and neck cancers, multidisciplinary strategy should be used, the treatment choice for head and neck cancers is based on the site or extension of the primary tumor [2].

In different combinations, surgery, radiation, and chemotherapy are used to manage HNC. Early-stage disease (stage I and stage II) is generally handled with surgery or radiation alone. Treatment involves platinum-based chemo radiation, with or without induction chemotherapy (IC) as a sequential therapy for most patients with locally advanced disease (stage III and IVA/B [2]).

For locally advanced head neck SCC chemoradiotherapy produces compara- 
ble results to surgery and radiotherapy but at the expense of side effects. However, parotid (PG) and Submandibular gland toxicity leads to loss of gland function [14].

Xerostomia is the most prominent complication in patients with head and neck carcinoma after radiotherapy (RTH) because radiation fields often involve bilaterally salivary glands, which are particularly radiosensitive [15].

Radiation-induced xerostomia is usually followed by impaired saliva processing and consequent co-morbidities such as oral infections, dental caries, loss of taste and dysphagia, which considerably decrease the quality of life of long-term RTH survivor [15].

It is proposed that diffusion-weighted magnetic resonance imaging (DW-MRI) is a promising procedure for evaluating changes due to radiotherapy. Our objective was to define the impact of RT/CRT on salivary gland before and after treatment s using DW-MRI [15].

The present study enrolled 43 patients with head and neck squamous cell carcinoma treated with definitive radiotherapy, sequential therapy or concurrent chemoradiotherapy at Clinical Oncology and Nuclear Medicine \& MRI diffusion scans were done at Diagnostic Radiology Departments Tanta University Hospitals throughout the period from May 2016 to May 2019.

AS regard xerostomia we characterized the distribution of ADC values in the parotid and submandibular glands at rest and in a stimulated state before and after RT. In the current study results showed that gustatory stimulation and radiation both changed the ADC values of the parotid and submandibular glands. In the present study, The SMGs received a mean dose of $39.86+28.22 \mathrm{~Gy} \&$ the mean dose to the entire RT \& LT parotid glands was $34.67 \mathrm{~Gy}$ and $33.90 \mathrm{~Gy}$ respectively. Our results were in harmony with that of Doornaert et al., 2015 who reported that the mean dose to the entire RT \& LT parotid glands was $43.9 \mathrm{~Gy}$ and 31.4 Gy respectively and the SMGs received a mean dose of $59.4-73.2 \mathrm{~Gy}$ [16].

The histological composition of submandibular glands is different from parotid glands. At resting state, they contribute to about $70 \%$ of all saliva. The severity of xerostomia is influenced markedly by hypo function of submandibular gland. Therefore, role of IMRT in sparing them should be evaluated [4].

- Parotid glands (PG):

According to site, when comparing the PG ROIs, in all time periods, including prior to treatment, there was a difference in ADC results, with the lower and deep ROIs having higher ADC values than the upper ROIs and this is consistent with Doornaert et al., 2015 [16]. We found that in pre-stimulation, Post-RTH ADC values were significantly higher than Pre-RTH values $(\mathrm{p}<0.001)$ and this is consistent with Doornaert et al., 2015 [16].

Post-stimulation, we found that Post-RTH ADC values of PGs were higher than at rest and this was significant only for LT upper lobe of PG (p: 0.009*) and this is consistent with Zhang et al., 2013 [14]. In the current study at rest, we 
found that ADC values were higher in pre-RTH than pre-chemotherapy. However it isn't statistically significant except for LT lower lobe. ADC change of PGs was higher in patients with T3 N2 hypopharyngeal carcinoma who received sequential therapy and it was statistically significant.

- Submandibular glands:

Post-RTH ADC values of SMGs at rest were significantly higher than pre-RTH values $(\mathrm{p}<0.001$ and this is consistent with Doornaert et al., 2015. Post-stimulation, we found that post-RTH ADC values of SMGs were significantly higher than at rest (p: 0.004) this is consistent with Doornaert et al., 2015. In the present study at rest, we found that pre-RTH ADC values of SMGs were higher than pre-chemotherapy values. However it isn't significant. ADC changes of SMGs were higher in patients with T3 N1, 2 oropharyngeal carcinoma who received sequential therapy and it was statistically significant. In all time settings, parotid gland ADC values were less than the SMG and this was consistent with Doornaert et al., 2015 [16].

According to Zhang et al., 2018 for parotid gland, it was shown that the loss of gland function was found to increase and the minimal gland hypofunnction was reported at $<10$ - 15 Gy mean dose. At radiation dose of 20 - 40 Gy there was gradual increase in loss of gland function. At doses greater than $75 \mathrm{~Gy}$, the loss of gland function reached up to $75 \%$ [4].

However overall response in sequential \& CCRTH groups was higher than the group of definitive RTH, xerostomia was developed in sequential \& CCRTH groups more than that of definitive RTH.

According to Zhang et al., 2018 xerostomia improved over time after IMRT, the potential benefits gained from IMRT were not apparent until 6 months or more after therapy [4]. As regard xerostomia questionnaire, $65 \%$ of patients experienced moderated to sever degrees of xerostomia similar to Darwis et al., 2017 who reported that $65 \%$ of patients had xerostomia According to xerostomia related quality of life scale questionnaire, $76.9 \%$ of patients who had xerostomia had low quality of life while $85.7 \%$ of patients who aren't experiencing xerostomia had high quality of life in compared to Darwis et al., 2017 who reported that $57.7 \%$ of patients who had xerostomia had low quality of life while $87 \%$ of patients who aren't experiencing xerostomia had high quality of life [17].

Cross-tabulation between xerostomia on the quality of life among head and neck cancer patients undergoing radiotherapy showed $\mathrm{p}=0.028$ means that there was a significant relationship between xerostomia and quality of life.

\section{Conclusions}

Diffusion weighted MRI is a non-invasive procedure for evaluating the function of the salivary gland in patients with radiation-induced xerostomia. As regard treatment modalities. ADC changes in both PGs \& SMGs were significantly higher in patients who received sequential therapy. ADC values for the PG were lower than the SMG at all time ADC change of PGs was higher in hypopharyngeal car- 
cinoma, while ADC change of SMGs higher in oropharyngeal carcinoma.ADC change in PGs \& SMGs was higher in T3, N1 \& N2.

Xerostomia was significantly related to the quality of life. Xerostomia questionnaire is a useful tool for assessment of quality of life for patients with radiation induced xerostomia.

\section{Recommendation}

- Submandibular gland sparing can reduce the risk of xerostomia.

- New modalities of radiotherapy are suggested, such as IMRT, IT has an important and efficient role in decreasing doses to salivary glands and consequently xerostomia decreases.

- A comparative study is recommended between sequential or CCRTH with IMRT versus 3D conformal definitive RTH with evaluation of xerostomia.

- Large sample size is recommended for better evaluation of xerostomia.

\section{Conflicts of Interest}

The authors declare no conflicts of interest regarding the publication of this paper.

\section{References}

[1] Siegel, R., Miller, K. and Jemal, A. (2017) Cancer Statistics, 2017. CA: A Cancer Journal for Clinicians, 67, 7-30. https://doi.org/10.3322/caac.21387

[2] Al-Saleh, K., Eid, R., Haddad, F.G.H., Saleh, N.K. and Kourie, R. (2018) New Developments in the Management of Head and Neck Cancer-Impact of Pembrolizumab. Therapeutics and Clinical Risk Management, 14, 295-303. https://doi.org/10.2147/TCRM.S125059

[3] Shavi, G.R., Thakur, B., Bhambal, A., Jain, S., Singh, V. and Shukla, A. (2015) Oral Health Related Quality of Life in Patients of Head and Neck Cancer Attending Cancer Hospital of Bhopal City, India. Journal of International Oral Health, 7, 21-27.

[4] Zhang, Y., Ou, D., Gu, Y., He, X. and Peng, W. (2018) Evaluation of Salivary Gland Function Using Diffusion-Weighted Magnetic Resonance Imaging for Follow-up of Radiation-Induced Xerostomia. Korean Journal of Radiology, 19, 758-766. https://doi.org/10.3348/kjr.2018.19.4.758

[5] Jingjiao, L., Huang, P., Changsheng, M., Yue, Z., Jinhu, C., Yueqiang, L., et al. (2018) Parotid Gland Radiation Dose Xerostomia Relationships Based on Actual Delivered Dose for Nasopharyngeal Carcinoma. The Journal of Applied Clinical Medical Physics, 19, 251-260. https://doi.org/10.1002/acm2.12327

[6] Zhang, Q., Wei, Y.M., Qi, Y.G. and Li, B.S. (2018) Early Changes in Apparent Diffusion Coefficient for Salivary Glands during Radiotherapy for Nasopharyngeal Carcinoma Associated with Xerostomia. Korean Journal of Radiology, 19, 328-333. https://doi.org/10.3348/kjr.2018.19.2.328

[7] Loimu, V., Seppälä, T., Kapanen, M., Tuomikoski, L., Nurmi, H., Mäkitie, Tenhunen, M., et al. (2017) Diffusion-Weighted Magnetic Resonance Imaging for Evaluation of Salivary Gland Function in Head and Neck Cancer Patients Treated with Intensity-Modulated Radiotherapy. Radiotherapy and Oncology, 122, 178-184. 
https://doi.org/10.1016/j.radonc.2016.07.008

[8] Xu, X.Q., Choi, Y.J., Sung, Y.S., Yoon, R.G., Jang, S.W., Park, J.E., et al. (2016) Intravoxel Incoherent Motion MR Imaging in the Head and Neck: Correlation with Dynamic Contrast-Enhanced MR Imaging and Diffusion-Weighted Imaging. Korean Journal of Radiology, 17, 641-649. https://doi.org/10.3348/kjr.2016.17.5.641

[9] Amid, M.B. (2017) Part II Head and Neck in: AJCC Cancer Staging Manual. 8th Edition, Springer, New York.

[10] Atikson, T.M., Ryan, S., Bennett, A.V., Stover, A.M., Saracino, R.M., Rogak, L.J., et al. (2016) The Association between Cinicia Based Common Terminology Criteria Adverse Events (CTACE) and Patient Reported Outcomes (PRO_0: A Systematic Review). Support Care Cancer, 24, 3669. https://doi.org/10.1007/s00520-016-3297-9

[11] Cox, J.D., Stetz, J. and Pajak, T.F. (1995) Toxicity Criteria of the Radiation Therapy Oncology (RTOG) and the European Organii: 2525-34zation for Research and Treatment of Cancer (EORTC). International Journal of Radiation Oncology, Biology, Physics, 31, 1341-1346. https://doi.org/10.1016/0360-3016(95)00060-C

[12] Drix, P., Nutys, S. and Van Den Bogaert, W. (2006) Radiation Induced Xerostomia in Patients with Head and Neck Cancer: A Literature Review. Cancer, 7, 2525-2534. https://doi.org/10.1002/cncr.22302

[13] IBM SPSS Data Collection Diveristiure.

[14] Jaume, M., Lluís Brunet, L., Eduard Lahor, S., et al. (2015) Salivary Secretory Disorders, Inducing Drugs, and Clinical Management. International Journal of Medical Sciences, 12, 811-824. https://doi.org/10.7150/ijms.12912

[15] Zhang, Y., Ou, D., Gu, Y., He, X., Peng, W., Mao, J., et al. (2013) Diffusion-Weighted MR Imaging of Salivary Glands with Gustatory Stimulation: Comparison before and after Radiotherapy. Acta Radiologica, 54, 928-933. https://doi.org/10.1177/0284185113491089

[16] Doornaert, P., Dahele, M., Ljumanovic, R., Bree, R., Slotman, B.J. and Castelijns, J.A. (2015) Use of Diffusion-Weighted Magnetic Resonance Imaging (DW-MRI) to Investigate the Effect of Chemoradiotherapy on the Salivary Glands. Acta Oncologica, 54, 1068-1071. https://doi.org/10.3109/0284186X.2014.987357

[17] Darwis, A.F., Hasibuan, S. and Tarigan, H.N. (2017) Effect Ofxerostomia on Quality of Life in Head and Neck Cancer Patients Undergoing Radiotherapy at RSUP H. Adam Malik Medan. Advances in Health Sciences Research, 8, 11-15. 


\section{Abbreviations}

ADC: Apparent diffusion coefficient;

CCRTH: concurrent chemoradiotherapy;

DW-MRI: diffusion, weighted magnetic resonance image;

HNSCC: head and neck squamous cell carcinoma;

NCI-CTC: national cancer institute-common toxicity criteria;

PG: parotid gland;

ROI: region of interest;

RTH: radiotherapy;

SGS: salivary gland scintigraphy;

SMG: submandibular salivary gland. 